Article

\title{
Synthesis, Characterization and Microwave-Promoted Catalytic Activity of Novel $N$-phenylbenzimidazolium Salts in Heck-Mizoroki and Suzuki-Miyaura Cross-Coupling Reactions under Mild Conditions
}

\section{Ülkü Yılmaz ${ }^{1}$, Hasan Küçükbay ${ }^{2, *}$, Selma Deniz ${ }^{3}$ and Nihat Şireci ${ }^{4}$}

1 Battalgazi Vocational School, İnönü University, Battalgazi, 44210 Malatya, Turkey; E-Mail: ulku.yilmaz@inonu.edu.tr

2 Department of Chemistry, Faculty of Science, İnönü University, 44280 Malatya, Turkey

3 Department of Elementary Education, Faculty of Education, Hakkari University, 30000 Hakkari, Turkey; E-Mail: selmadeniz@hakkari.edu.tr

4 Department of Elementary Education, Faculty of Education, Adiyaman University, 02040 Adiyaman, Turkey; E-Mail: nsireci@adiyaman.edu.tr

* Author to whom correspondence should be addressed; E-Mail: hasan.kucukbay@inonu.edu.tr; Tel.: +90-422-377-3881; Fax: +90-422-341-0037.

Received: 25 December 2012; in revised form: 29 January 2013 / Accepted: 16 February 2013 / Published: 25 February 2013

\begin{abstract}
A number of novel benzimidazolium salts having aryl substituents such as $N$-phenyl, 4-chlorophenyl and various alkyl substituents were synthesized. Their microwave-assisted catalytic activities were evaluated in Heck-Mizoroki and Suzuki-Miyaura cross-coupling reactions using a catalytic system consisting of $\mathrm{Pd}(\mathrm{OAc})_{2} / \mathrm{K}_{2} \mathrm{CO}_{3}$ in $\mathrm{DMF} / \mathrm{H}_{2} \mathrm{O}$ under mild reaction conditions with consistent high yields, except those of 2-bromopyridine.
\end{abstract}

Keywords: $N$-phenylbenzimidazoles; Suzuki-Miyaura reaction; Heck-Mizoroki reaction; microwaves; NHC-precursor

\section{Introduction}

In recent years, palladium catalyzed the $\mathrm{C}-\mathrm{C}$ bond forming reaction of aryl halides with phenylboronic acid and styrene has been great interest for the synthesis of agrochemicals, 
pharmaceuticals and advanced enantioselective synthesis of natural products $[1,2]$. These ingenious carbon-carbon bond formation methods, the Heck-Mizoroki and Suzuki-Miyaura methods, also play vital roles in the development of new generation organic materials with novel electronic, optical or mechanical properties [3]. The Heck-Mizoroki and Suzuki-Miyaura reactions are most commonly used as model reactions to evaluate and compare the catalytic activity of metal complexes, to highlight ligand effects, and to understand the role of solvent and other additives [4-8]. Nevertheless, most efficient Heck-Mizoroki and Suzuki-Miyaura reactions reported were carried out in organic solvents with phosphine-based compounds as the ancillary ligand. In order to overcome the toxicity, air and/or moisture-sensitivity of phosphine-based ligands, many efforts have been made to find out alternative ligands [9-12]. In this context, $N$-heterocyclic carbenes (NHCs) are promising alternative ligands in palladium-catalyzed cross-coupling reactions due to their strong $\sigma$-donor but poor $\pi$-acceptor abilities, low toxicity, stability to air, moisture and heating. They are considered alternatives to phosphine ligands in metal complexes. For this reason, the design and synthesis of novel and effective NHCs have attracted a great deal of attention from both academia and industry [13-17]. Furthermore, ligandfree palladium catalyzed $\mathrm{C}-\mathrm{C}$ coupling reactions were also studied in water [18-22].

On the other hand, the application of microwave irradiation for promoting reactions has become a versatile tool in organic synthesis. Rapid heating, reduced reaction times, and in many cases, increased yields have made microwave assisted organic synthesis a commonly used tool, especially for preparative medicinal chemistry. The shortened reaction times offered by microwave heating indicate that additional benefits could be obtained from an energy-saving perspective by exploiting this technology even further [23-25]. For this reason, microwave-promoted synthesis is an area of increasing interest in both academic and industrial laboratories. The use of metal catalysts in conjunction with microwaves may have significant advantages over traditional heating methods since the inverted temperature gradient under microwave conditions may lead to an increased lifetime of catalyst through the elimination of wall effects [26]. There are extensive studies about Heck-Mizoroki and Suzuki-Miyaura type $\mathrm{C}-\mathrm{C}$ cross-coupling reactions incorporating microwave irradiation using various ligands other than the benzimidazole moiety [22,27-35].

During recent decades, many researchers have focused mainly on the development of milder reaction conditions using new catalysts and reaction systems. In order to find a more efficient palladium catalyst, we also synthesized a series of some new benzimidazole salts, containing alkyl or heterocycle substituted alkyl and bis-benzimidazole salts as a NHC ligand and we tested the activity of Pd-NHC based catalytic systems prepared in-situ for the Heck-Mizoroki and Suzuki-Miyaura cross-coupling reactions under microwave heating conditions [36-40].

In continuation of this subject, herein we describe the synthesis of new benzimidazole salts 1-10 containing phenyl or 4-chlorophenyl on one nitrogen atom of the benzimidazole ring and an alkyl or substituted alkyl on the other nitrogen atom. The compounds were fully characterized by elemental analysis, IR, ${ }^{13} \mathrm{C}-\mathrm{NMR}$, and ${ }^{1} \mathrm{H}-\mathrm{NMR}$ spectroscopy. We also report the microwave-assisted catalytic activity of $\mathrm{Pd}(\mathrm{OAc})_{2} /$ base/novel benzimidazoles catalytic system in Heck-Mizoroki and SuzukiMiyaura cross-coupling reactions. 


\section{Results and Discussion}

1-Phenylbenzimidazole and 1-(4-chlorophenyl)benzimidazole were synthesized from $N$-phenyl-1,2diaminobenzene and $\mathrm{N}$-(4-chlorophenyl)-1,2-diaminobenzene by reaction with formic acid in $4 \mathrm{~N} \mathrm{HCl}$ according to the Phillips' method, respectively [41,42].

Novel benzimidazolium salts, 1-10 were prepared in good yields of $70 \%-92 \%$ by treatment of 1-phenylbenzimidazole or 1-(4-chlorophenyl)benzimidazole with appropriate alkyl halides in refluxing DMF. The synthesis of the benzimidazolium salts $\mathbf{1 - 1 0}$ is summarized in Scheme 1.

Scheme 1. Synthesis of $N$-arylbenzimidazole derivatives.
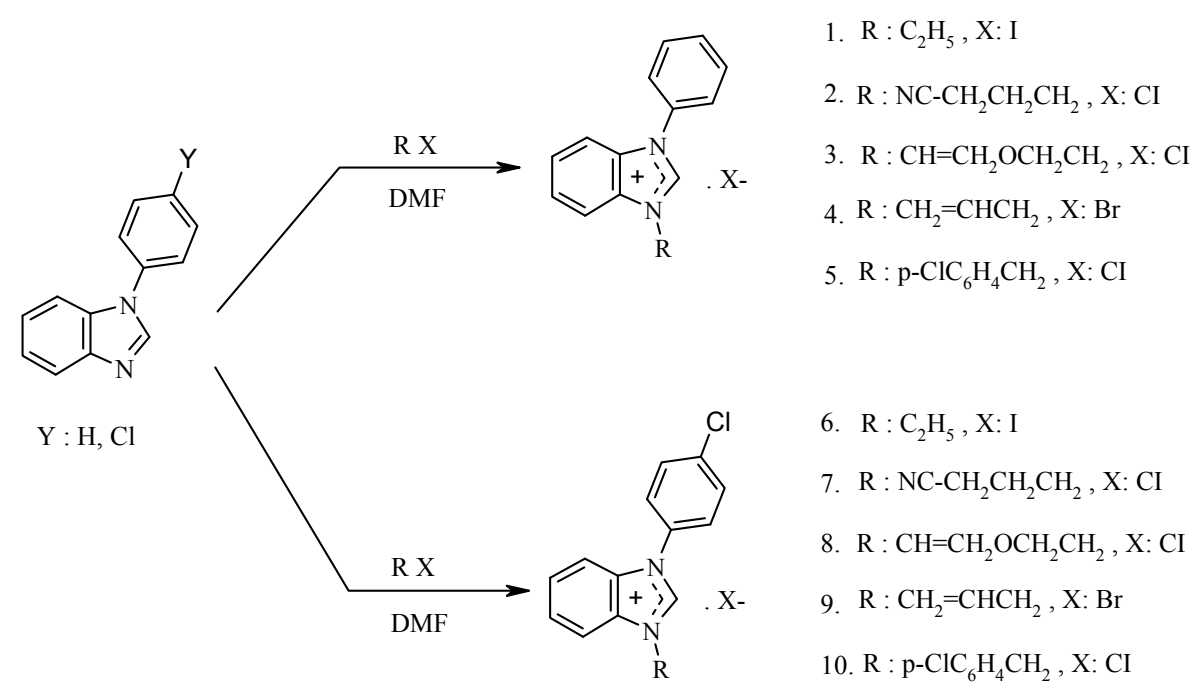

The benzimidazolium salts are air and moisture-stable both in the solid state and in solution. The new benzimidazole derivatives 1-10 were characterized by ${ }^{1} \mathrm{H}-\mathrm{NMR},{ }^{13} \mathrm{C}\left\{{ }^{1} \mathrm{H}\right\} \mathrm{NMR}$, IR and elemental analysis techniques, which support the proposed structures. The value of $\delta\left[{ }^{13} \mathrm{C}\left\{{ }^{1} \mathrm{H}\right\}\right]$, $\mathrm{NCH}_{2} \mathrm{Ph}$ in benzimidazolium salts $\mathbf{5}$ and $\mathbf{1 0}$ were both found to be $49.9 \mathrm{ppm}$. The benzylic protons signals for the benzimidazolium salts $\mathbf{5}$ and $\mathbf{1 0}$ were found to be 5.89 and $5.92 \mathrm{ppm}$, respectively. The value of $\delta\left[{ }^{13} \mathrm{C}\left\{{ }^{1} \mathrm{H}\right\}\right], \mathrm{NCHN}$ in benzimidazolium salts is usually around $142 \pm 4$ [37]. For benzimidazolium salts, 1-10 it was found to be 142.8, 143.6, 143.3, 143.3, 143.6, 142.9, 143.7, 143.5, 143.4 and $143.7 \mathrm{ppm}$, respectively. These values are in good agreement with the previously reported results [10]. The $\mathrm{NCHN}$ proton signals for the benzimidazolium salts, 1-10 were observed as singlets at $10.20,10.74,10.32,10.28,10.51,10.18,10.51,10.30,10.34$ and 10.67 ppm, respectively. As expected, the highest downfield shifts of the $\mathrm{NCHN}$ proton signals were observed among the benzimidazolium salts which bear electron withdrawing chloride and cyanide substituents on the nitrogen atom of the benzimidazole scaffold. Thus, the hydrogen atom on the 2-position of the benzimidazolium salts behave as acids in the sense that they give up protons to suitably strong bases. As a result of acidic proton of these type benzimidazolium salts, electron-rich olefins can be synthesized easily, as shown in Scheme 2 [43-45]. The carbon-nitrogen band frequencies, $v_{(\mathrm{C}=\mathrm{N})}$ for benzimidazole salts, $\mathbf{1}-\mathbf{1 0}$ were observed at 1560, 1557, 1558, 1554 and 1558, 1559, 1557, 1557, 1552 and $1554 \mathrm{~cm}^{-1}$, respectively. 
Scheme 2. Electron-rich olefin synthesis through the acidic hydrogen atom removing on the 2-position of the benzimidazole.

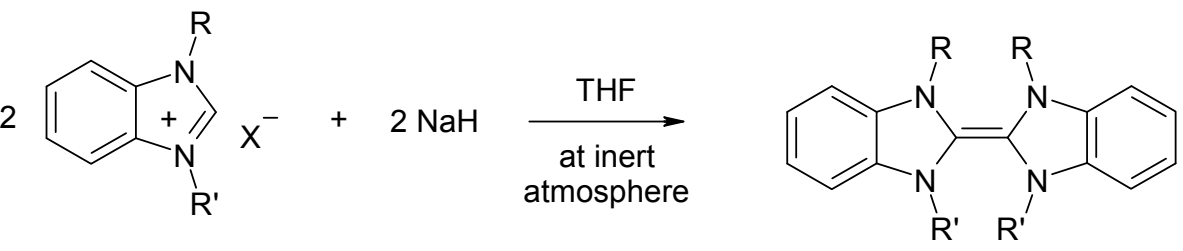

$\mathrm{R}, \mathrm{R}^{\prime}=$ alkyl or aryl

\subsection{The Heck-Mizoroki Coupling Reaction}

The Heck-Mizoroki coupling is one of the most studied $\mathrm{C}-\mathrm{C}$ bond forming reactions between alkenes and aromatic rings and is widely used by both academic and industrial laboratories. The industrial applications of this reaction can be observed in the fine chemical field, such as in the manufacture of pharmaceuticals and herbicides [46-48]. The catalytic yield of the Heck-Mizoroki coupling reaction is dependent on a variety of parameters such as temperature, solvent, base and nature of the catalyst and catalyst loading. For this reason, the optimum reaction parameters were investigated before starting the series of coupling experiments.

In order to find the optimum reaction conditions for the Heck-Mizoroki coupling reaction, a series of experiments was performed with 2-bromopyridine and styrene as model compounds. The test reactions were performed using different bases such as $\mathrm{K}_{2} \mathrm{CO}_{3}, \mathrm{Cs}_{2} \mathrm{CO}_{3}, \mathrm{Et}_{3} \mathrm{~N}$ and $\mathrm{DBU}$ (1,8-diazabicyclo[5.4.0]undec-7-ene) and different solvents such as $\mathrm{DMF} / \mathrm{H}_{2} \mathrm{O}, \mathrm{EtOH} / \mathrm{H}_{2} \mathrm{O}, \mathrm{DMA}$ and $\mathrm{C}_{2} \mathrm{H}_{4}(\mathrm{OH})_{2} / \mathrm{H}_{2} \mathrm{O}$ for different times and temperatures. It was found that the Heck-Mizoroki coupling reaction catalyzed by $\mathbf{1}, \mathrm{Pb}(\mathrm{OAc})_{2}$ and base catalyst system gave the highest yield when using $\mathrm{DMF} / \mathrm{H}_{2} \mathrm{O}$ mixture as a solvent and $\mathrm{K}_{2} \mathrm{CO}_{3}$ or $\mathrm{Cs}_{2} \mathrm{CO}_{3}$ as a base at 80 and $100{ }^{\circ} \mathrm{C}$ microwave heating in $10 \mathrm{~min}$. Very little improvement was observed for the catalytic yields upon increasing the temperature from $80{ }^{\circ} \mathrm{C}$ to $100{ }^{\circ} \mathrm{C}$. No considerable increase in catalytic reaction yields was observed by prolonging the time from 10 to 20 min either. After these results, we chose $\mathrm{K}_{2} \mathrm{CO}_{3}$ as a base, being cheaper than $\mathrm{Cs}_{2} \mathrm{CO}_{3}$, water/DMF as a solvent and $80{ }^{\circ} \mathrm{C}$ as reaction temperature for the lower energy consumption than at $100{ }^{\circ} \mathrm{C}$. We also tested the catalytic yields using a conventional heating system in a preheated oil bath 5,10 and $20 \mathrm{~min}$ at $60{ }^{\circ} \mathrm{C}$ and $80{ }^{\circ} \mathrm{C}$, but the yields of the catalytic conversions were either nil or very low (Table 1, entries 1-6). Control experiments showed that the yield of Heck-Mizoroki coupling reaction were decreased in the absence of $\mathbf{1}$ in 10 min under microwave heating (Table 1, entries 16,17). Use of a higher amounts of benzimidazolium salt (4 mol \%) did not improve the catalytic yield (Table 1, entry 18). The coupling reaction did not occur in the absence of $\mathrm{Pd}(\mathrm{OAc})_{2}$ after $10 \mathrm{~min}$ under microwave heating (Table 1, entry 19). The test experiments results for optimization of the Heck-Mizoroki coupling reaction are given in Table 1. After optimization experiments (Table 1), we found that use of the catalytic system consists of $1 \% \mathrm{~mol} \mathrm{Pd}(\mathrm{OAc})_{2}, 2 \% \mathrm{~mol}$ of 1-10 and 2 mol K${ }_{2} \mathrm{CO}_{3}$ in $\mathrm{DMF} / \mathrm{H}_{2} \mathrm{O}(1: 1)$ at $80{ }^{\circ} \mathrm{C} / 300 \mathrm{~W}$ microwave heating led to the best conversion within $10 \mathrm{~min}$. 
Table 1. Test experiments for optimization of the Heck-Mizoroki coupling reactions.

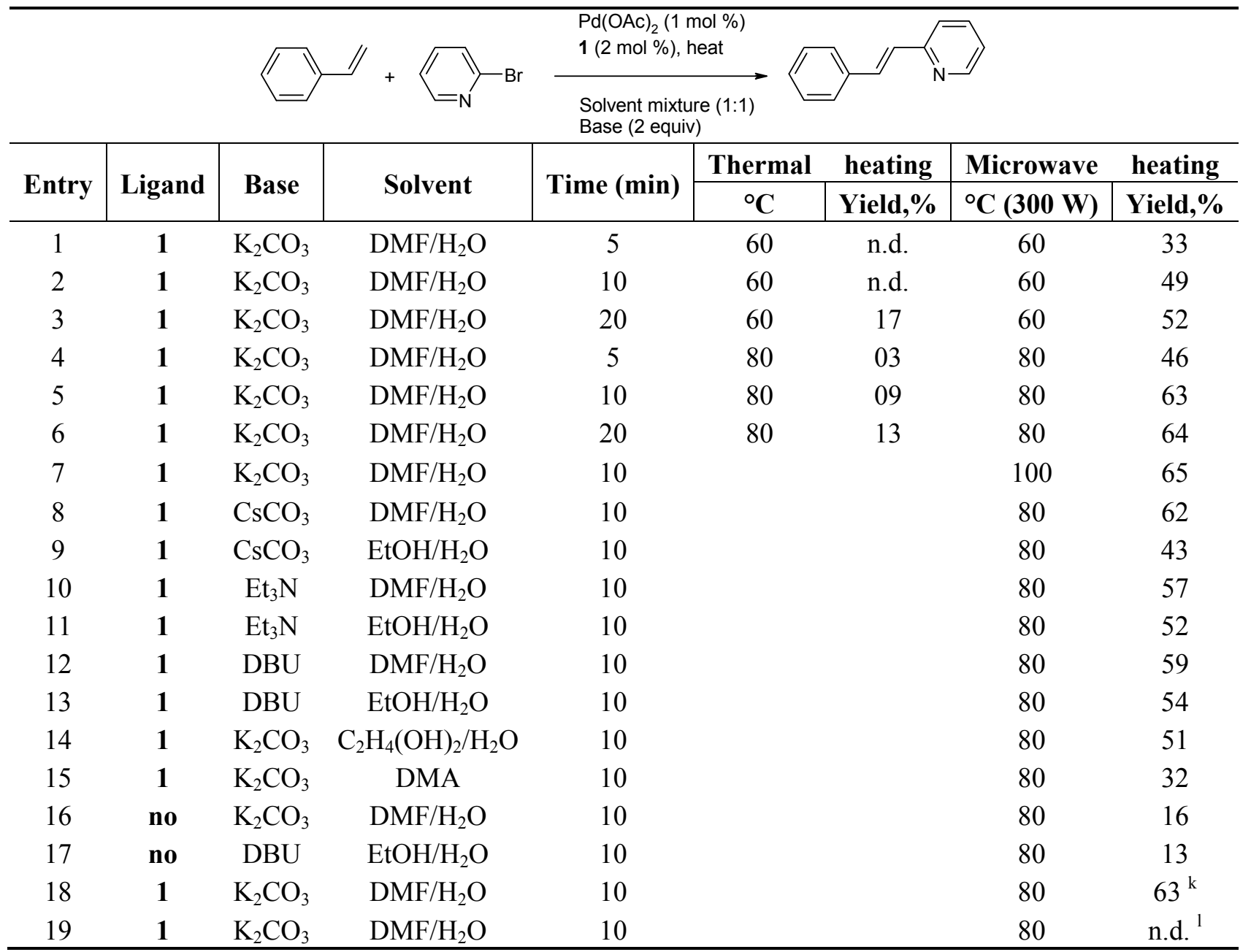

n.d.: not detected. Reaction conditions are same as indicated in the text. Yields are based on aryl bromide.

Reactions were monitored by GC-MS. 4 mol \% of $1^{k}$, without $\mathrm{Pd}(\mathrm{OAc})_{2}{ }^{1}$.

Using the above optimized reaction conditions, the coupling reactions of four different aryl halides (bearing electron-donating, electron-withdrawing groups and 2-bromopyridine) and styrene were then investigated. The results are shown in Table 2. Among the aryl halides, the catalytic yield of those involving 2-bromopyridine were found to be moderate but the others gave high yields.

Table 2. The Heck-Mizoroki coupling reactions of aryl halides with styrene.

\begin{tabular}{|c|c|c|c|c|c|}
\hline \multirow[b]{2}{*}{ Entry } & \multirow[b]{2}{*}{$\mathbf{R}$} & \multirow[b]{2}{*}{$\mathbf{Z}$} & \multicolumn{2}{|c|}{$\begin{array}{l}\mathrm{Pd}(\mathrm{OAC})_{2}(1 \mathrm{~mol} \%), \\
1-10(2 \mathrm{~mol} \%), \mathrm{mw}(300 \mathrm{~W}) \\
\mathrm{DMF}^{2} / \mathrm{H}_{2} \mathrm{O}(1: 1), 80^{\circ} \mathrm{C}, 10 \mathrm{~min} \\
\mathrm{~K}_{2} \mathrm{CO}_{3}(2 \text { equiv })\end{array}$} & \multirow[b]{2}{*}{ Conversion $^{a}(\%)$} \\
\hline & & & $\mathbf{X}$ & Salt & \\
\hline 1 & $\mathrm{H}$ & $\mathrm{N}$ & $\mathrm{Br}$ & 1 & $46^{\mathrm{b}}$ \\
\hline 2 & $\mathrm{H}$ & $\mathrm{N}$ & $\mathrm{Br}$ & 1 & $63^{\mathrm{c}}$ \\
\hline 3 & $\mathrm{H}$ & $\mathrm{N}$ & $\mathrm{Br}$ & 1 & $64^{\mathrm{d}}$ \\
\hline 4 & $\mathrm{H}$ & $\mathrm{N}$ & $\mathrm{Br}$ & 1 & $03^{\mathrm{e}}$ \\
\hline 5 & $\mathrm{H}$ & $\mathrm{N}$ & $\mathrm{Br}$ & 1 & $09^{\mathrm{f}}$ \\
\hline 6 & $\mathrm{H}$ & $\mathrm{N}$ & $\mathrm{Br}$ & no & $16^{\mathrm{g}}$ \\
\hline 7 & $\mathrm{H}$ & $\mathrm{CH}$ & I & 1 & 98 \\
\hline
\end{tabular}


Table 2. Cont.

\begin{tabular}{|c|c|c|c|c|c|}
\hline Entry & $\mathbf{R}$ & $\bar{Z}$ & $\mathbf{X}$ & Salt & Conversion $^{\mathrm{a}}(\%)$ \\
\hline 8 & $\mathrm{H}$ & $\mathrm{CH}$ & $\mathrm{I}$ & 2 & 98 \\
\hline 9 & $\mathrm{H}$ & $\mathrm{CH}$ & $\mathrm{I}$ & 3 & 97 \\
\hline 10 & $\mathrm{H}$ & $\mathrm{CH}$ & $\mathrm{I}$ & 4 & 96 \\
\hline 11 & $\mathrm{H}$ & $\mathrm{CH}$ & $\mathrm{I}$ & 5 & 96 \\
\hline 12 & $\mathrm{H}$ & $\mathrm{CH}$ & $\mathrm{I}$ & 6 & $9391^{\mathrm{i}}$ \\
\hline 13 & $\mathrm{H}$ & $\mathrm{CH}$ & $\mathrm{I}$ & 7 & $90^{\mathrm{i}}$ \\
\hline 14 & $\mathrm{H}$ & $\mathrm{CH}$ & $\mathrm{I}$ & 8 & $89^{\mathrm{i}}$ \\
\hline 15 & $\mathrm{H}$ & $\mathrm{CH}$ & $\mathrm{I}$ & 9 & $87^{\mathrm{i}}$ \\
\hline 16 & $\mathrm{H}$ & $\mathrm{CH}$ & $\mathrm{I}$ & 10 & $88^{\mathrm{i}}$ \\
\hline 17 & $\mathrm{OCH}_{3}$ & $\mathrm{CH}$ & $\mathrm{I}$ & 1 & 99 \\
\hline 18 & $\mathrm{OCH}_{3}$ & $\mathrm{CH}$ & I & 2 & 98 \\
\hline 19 & $\mathrm{OCH}_{3}$ & $\mathrm{CH}$ & I & 3 & 98 \\
\hline 20 & $\mathrm{OCH}_{3}$ & $\mathrm{CH}$ & I & 4 & 98 \\
\hline 21 & $\mathrm{OCH}_{3}$ & $\mathrm{CH}$ & I & 5 & 97 \\
\hline 22 & $\mathrm{OCH}_{3}$ & $\mathrm{CH}$ & I & 6 & $95^{\mathrm{i}}$ \\
\hline 23 & $\mathrm{OCH}_{3}$ & $\mathrm{CH}$ & I & 7 & $9489^{\mathrm{i}}$ \\
\hline 24 & $\mathrm{OCH}_{3}$ & $\mathrm{CH}$ & I & 8 & $90^{\mathrm{i}}$ \\
\hline 25 & $\mathrm{OCH}_{3}$ & $\mathrm{CH}$ & I & 9 & $91^{\mathrm{i}}$ \\
\hline 26 & $\mathrm{OCH}_{3}$ & $\mathrm{CH}$ & I & 10 & $90^{\mathrm{i}}$ \\
\hline 27 & $\mathrm{COCH}_{3}$ & $\mathrm{CH}$ & $\mathrm{Br}$ & 1 & 99 \\
\hline 28 & $\mathrm{COCH}_{3}$ & $\mathrm{CH}$ & $\mathrm{Br}$ & 2 & 99 \\
\hline 29 & $\mathrm{COCH}_{3}$ & $\mathrm{CH}$ & $\mathrm{Br}$ & 3 & 98 \\
\hline 30 & $\mathrm{COCH}_{3}$ & $\mathrm{CH}$ & $\mathrm{Br}$ & 4 & 95 \\
\hline 31 & $\mathrm{COCH}_{3}$ & $\mathrm{CH}$ & $\mathrm{Br}$ & 5 & 96 \\
\hline 32 & $\mathrm{COCH}_{3}$ & $\mathrm{CH}$ & $\mathrm{Br}$ & 6 & $94^{\mathrm{i}}$ \\
\hline 33 & $\mathrm{COCH}_{3}$ & $\mathrm{CH}$ & $\mathrm{Br}$ & 7 & $9692^{\mathrm{i}}$ \\
\hline 34 & $\mathrm{COCH}_{3}$ & $\mathrm{CH}$ & $\mathrm{Br}$ & 8 & $90^{\mathrm{i}}$ \\
\hline 35 & $\mathrm{COCH}_{3}$ & $\mathrm{CH}$ & $\mathrm{Br}$ & 9 & $87^{\mathrm{i}}$ \\
\hline 36 & $\mathrm{COCH}_{3}$ & $\mathrm{CH}$ & $\mathrm{Br}$ & 10 & $86^{\mathrm{i}}$ \\
\hline 37 & $\mathrm{H}$ & $\mathrm{N}$ & $\mathrm{Br}$ & 2 & 62 \\
\hline 38 & $\mathrm{H}$ & $\mathrm{N}$ & $\mathrm{Br}$ & 3 & 62 \\
\hline 39 & $\mathrm{H}$ & $\mathrm{N}$ & $\mathrm{Br}$ & 4 & 60 \\
\hline 40 & $\mathrm{H}$ & $\mathrm{N}$ & $\mathrm{Br}$ & 5 & 61 \\
\hline 41 & $\mathrm{H}$ & $\mathrm{N}$ & $\mathrm{Br}$ & 6 & 59 \\
\hline 42 & $\mathrm{H}$ & $\mathrm{N}$ & $\mathrm{Br}$ & 7 & 58 \\
\hline 43 & $\mathrm{H}$ & $\mathrm{N}$ & $\mathrm{Br}$ & 8 & 56 \\
\hline 44 & $\mathrm{H}$ & $\mathrm{N}$ & $\mathrm{Br}$ & 9 & 57 \\
\hline 45 & $\mathrm{H}$ & $\mathrm{N}$ & $\mathrm{Br}$ & 10 & 58 \\
\hline
\end{tabular}

${ }^{a}$ Conversions were determined by GC-MS based on the aryl halide. Reaction conditions: temperature ramped to $80{ }^{\circ} \mathrm{C}$ ( $\left.3 \mathrm{~min}\right)$ and held for $5^{\mathrm{b}} \mathrm{min}, 10^{\mathrm{c}} \mathrm{min}$ and $20^{\mathrm{d}} \mathrm{min}$. In preheated oil bath, $5^{\mathrm{e}} \mathrm{min}$ and $10^{\mathrm{f}} \mathrm{min}$ with thermal heating at $80{ }^{\circ} \mathrm{C}$. Temperature ramped to $80^{\circ} \mathrm{C}(3 \mathrm{~min})$ and held for $10^{\mathrm{g}} \mathrm{min}$ without salt (1). Isolated yields; ${ }^{\mathrm{i}}$ n.d., not detected. 
The benzimidazolium salts bearing an electron releasing ethyl substituent (1 and 6) are generally more effective than other salts examined. On the other hand, benzimidazole salts having a 4-chlorophenyl substituent on the nitrogen atom were generally slightly less reactive for the Heck-Mizoroki reaction than the corresponding phenyl substituent. Of the four different aryl halides used in the Heck-Mizoroki coupling with styrene, those with electron-withdrawing substituents (4-bromoacetphenone) were found to give the highest yield (Table 2, entries 27-36). Furthermore, aryl iodides, those with electron releasing methoxy substituents and without any substituent were also found to give better yield than 2-bromopyridine.

\subsection{The Suzuki-Miyaura Coupling Reaction}

Biaryls represent the mostly common structural motif in a broad range of susbtances, from naturally occurring potentially useful therapeutic agents to versatile building materials for light-emitting diodes, liquid crystals and many organic compounds with novel electronic, optical properties [49,50].

In order to find the optimum reaction conditions for the Suzuki coupling reaction, a series of experiments catalyzed couplings between 2-bromopyridine and phenylboronic acid as a model compounds were performed using similar parameters as in the Heck-Mizoroki coupling reactions described above. It was found that the Suzuki-Miyaura coupling reaction catalyzed by $\mathbf{1}, \mathrm{Pb}(\mathrm{OAc})_{2}$ and base catalyst system gave the highest yield when using $\mathrm{DMF} / \mathrm{H}_{2} \mathrm{O}$ mixture as a solvent and $\mathrm{K}_{2} \mathrm{CO}_{3}$ or $\mathrm{Cs}_{2} \mathrm{CO}_{3}$ as a base at $80{ }^{\circ} \mathrm{C}$ microwave heating in $10 \mathrm{~min}$. After these results, we chose $\mathrm{K}_{2} \mathrm{CO}_{3}$ as a base, being cheaper than $\mathrm{Cs}_{2} \mathrm{CO}_{3}$, water/DMF as a solvent and $80{ }^{\circ} \mathrm{C}$ as reaction temperature for the optimum reaction conditions. The conventional heating system in a preheated oil bath 5, 10 and 20 min at $60{ }^{\circ} \mathrm{C}, 80{ }^{\circ} \mathrm{C}$ and $100{ }^{\circ} \mathrm{C}$ was not appropriate for these conversions (Table 3, entries 1-9). When both microwave results and conventional preheated oil bath results were compared, we observed a clear improvement in yield and reaction time with microwave heating. Control experiments showed that the yield of Suzuki coupling reaction was decreased in the absence of $\mathbf{1}$ in 10 min under microwave heating (Table 3, entry 18). No coupling reaction was observed in the absence of $\mathrm{Pd}(\mathrm{OAc})_{2}$ after 10 min under microwave heating (Table 3, entry 20). The test experiments results for optimization of the Suzuki-Miyaura coupling reaction are given in Table 3.

After having established the optimized coupling reaction conditions (Table 3) the scope of the reaction and efficiencies of the benzimidazolium salts were evaluated by investigating the coupling of the phenylboronic acid with various aryl halides and a heteroaryl bromide. Under the optimized conditions, reaction of $p$-bromoacetophenone, $p$-iodoanisole and iodobenzene with phenylboronic acid gave almost identical high yields using a catalytic system consisting of $2 \mathrm{~mol} \%$ benzimidazole salt 1-10, $1 \mathrm{~mol} \% \mathrm{Pd}(\mathrm{OAc})_{2}$ and 2 equiv. $\mathrm{K}_{2} \mathrm{CO}_{3}$ in $\mathrm{DMF} / \mathrm{H}_{2} \mathrm{O}(1: 1)$ at $80{ }^{\circ} \mathrm{C}$ under microwave irradiation $(300 \mathrm{~W})$ within $10 \mathrm{~min}$. On the other hand, 2-bromopyridine gave a moderate yield using the optimized conditions. This is also good result considering the difficult synthesis of 2-arylpyridines using expensive organometallic reagents such as phenyl lithium or phenyl magnesium halides under an inert atmosphere. Of the four different aryl halides used in the Suzuki-Miyaura coupling with phenylboronic acid, those with electron-withdrawing substituents were found to give the highest yield (Table 4, entries 27-36). 
Table 3. Test experiments for optimization of the Suzuki-Miyaura coupling reactions.

\begin{tabular}{|c|c|c|c|c|c|c|c|c|}
\hline & & & $\mathrm{OH})_{2}+$ & $\begin{array}{l}\mathrm{Pd}(\mathrm{C} \\
1(2\end{array}$ & $\begin{array}{l}\mathrm{Ac})_{2}(1 \mathrm{~mol} \%) \\
\text { nol \%), heat } \\
\text { nt mixture (1:1 } \\
(2 \text { equiv) }\end{array}$ & 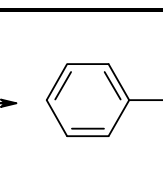 & $\overline{1}$ & \\
\hline Entry & Ligand & Base & Solvent & Time (min) & Thermal & heating & Microwave & heating \\
\hline & & & & & ${ }^{\circ} \mathrm{C}$ & Yield,\% & ${ }^{\circ} \mathrm{C}(300 \mathrm{~W})$ & Conver. $^{\text {a }}, \%$ \\
\hline 1 & 1 & $\mathrm{~K}_{2} \mathrm{CO}_{3}$ & $\mathrm{DMF} / \mathrm{H}_{2} \mathrm{O}$ & 5 & 60 & 0 & 60 & 47 \\
\hline 2 & 1 & $\mathrm{~K}_{2} \mathrm{CO}_{3}$ & $\mathrm{DMF} / \mathrm{H}_{2} \mathrm{O}$ & 10 & 60 & 4 & 60 & 53 \\
\hline 3 & 1 & $\mathrm{~K}_{2} \mathrm{CO}_{3}$ & $\mathrm{DMF} / \mathrm{H}_{2} \mathrm{O}$ & 20 & 60 & 9 & 60 & 55 \\
\hline 4 & 1 & $\mathrm{~K}_{2} \mathrm{CO}_{3}$ & $\mathrm{DMF} / \mathrm{H}_{2} \mathrm{O}$ & 5 & 80 & 6 & 80 & 67 \\
\hline 5 & 1 & $\mathrm{~K}_{2} \mathrm{CO}_{3}$ & $\mathrm{DMF} / \mathrm{H}_{2} \mathrm{O}$ & 10 & 80 & 11 & 80 & 75 \\
\hline 6 & 1 & $\mathrm{~K}_{2} \mathrm{CO}_{3}$ & $\mathrm{DMF} / \mathrm{H}_{2} \mathrm{O}$ & 20 & 80 & 13 & 80 & 76 \\
\hline 7 & 1 & $\mathrm{~K}_{2} \mathrm{CO}_{3}$ & $\mathrm{DMF} / \mathrm{H}_{2} \mathrm{O}$ & 5 & 100 & 7 & 100 & 67 \\
\hline 8 & 1 & $\mathrm{~K}_{2} \mathrm{CO}_{3}$ & $\mathrm{DMF} / \mathrm{H}_{2} \mathrm{O}$ & 10 & 100 & 11 & 100 & 76 \\
\hline 9 & 1 & $\mathrm{~K}_{2} \mathrm{CO}_{3}$ & $\mathrm{DMF} / \mathrm{H}_{2} \mathrm{O}$ & 20 & 100 & 14 & 100 & 77 \\
\hline 10 & 1 & $\mathrm{CsCO}_{3}$ & $\mathrm{DMF} / \mathrm{H}_{2} \mathrm{O}$ & 10 & & & 80 & 75 \\
\hline 11 & 1 & $\mathrm{CsCO}_{3}$ & $\mathrm{EtOH} / \mathrm{H}_{2} \mathrm{O}$ & 10 & & & 80 & 66 \\
\hline 12 & 1 & $\mathrm{~K}_{2} \mathrm{CO}_{3}$ & $\mathrm{H}_{2} \mathrm{O}$ & 10 & & & 80 & 39 \\
\hline 13 & 1 & $\mathrm{~K}_{2} \mathrm{CO}_{3}$ & $\mathrm{C}_{2} \mathrm{H}_{4}(\mathrm{OH})_{2} / \mathrm{H}_{2} \mathrm{O}$ & 10 & & & 80 & 56 \\
\hline 14 & 1 & $\mathrm{~K}_{2} \mathrm{CO}_{3}$ & DMA & 10 & & & 80 & 47 \\
\hline 15 & 1 & DBU & $\mathrm{DMF} / \mathrm{H}_{2} \mathrm{O}$ & 10 & & & 80 & 64 \\
\hline 16 & 1 & DBU & $\mathrm{EtOH} / \mathrm{H}_{2} \mathrm{O}$ & 10 & & & 80 & 66 \\
\hline 17 & 1 & $\mathrm{~K}_{2} \mathrm{CO}_{3}$ & Glycerine $/ \mathrm{H}_{2} \mathrm{O}$ & 10 & & & 80 & 58 \\
\hline 18 & no & $\mathrm{K}_{2} \mathrm{CO}_{3}$ & $\mathrm{DMF} / \mathrm{H}_{2} \mathrm{O}$ & 10 & & & 10 & 32 \\
\hline 19 & 1 & $\mathrm{~K}_{2} \mathrm{CO}_{3}$ & $\mathrm{DMF} / \mathrm{H}_{2} \mathrm{O}$ & 10 & & & 10 & $74^{\mathrm{m}}$ \\
\hline 20 & 1 & $\mathrm{~K}_{2} \mathrm{CO}_{3}$ & $\mathrm{DMF} / \mathrm{H}_{2} \mathrm{O}$ & 10 & & & 10 & n.d. ${ }^{n}$ \\
\hline 21 & 1 & $\mathrm{~K}_{2} \mathrm{CO}_{3}$ & $\mathrm{DMF} / \mathrm{H}_{2} \mathrm{O}$ & 10 & & & 10 & $77^{p}$ \\
\hline 22 & 1 & $\mathrm{~K}_{2} \mathrm{CO}_{3}$ & $\mathrm{DMF} / \mathrm{H}_{2} \mathrm{O}$ & 10 & & & 10 & $65^{\mathrm{r}}$ \\
\hline
\end{tabular}

${ }^{\mathrm{a}}$ Conversions were determined by GC-MS based on the aryl halide. 4 mol $\%$ of $1^{\mathrm{m}}$, without $\operatorname{Pd}(\mathrm{OAc})_{2}{ }^{\mathrm{n}}$, $2 \mathrm{~mol} \%$ of $\mathrm{Pd}(\mathrm{OAc})_{2}{ }^{\mathrm{p}}, 0.5 \mathrm{~mol} \%$ of $\mathrm{Pd}(\mathrm{OAc})_{2}{ }^{\mathrm{r}}$.

Table 4. The Suzuki-Miyaura coupling reactions of aryl halides with phenylboronic acid.

\begin{tabular}{|c|c|c|c|c|c|}
\hline \multirow[b]{2}{*}{ Entry } & \multirow[b]{2}{*}{$\mathbf{R}$} & \multirow[b]{2}{*}{$\mathbf{Z}$} & \multicolumn{2}{|c|}{$\begin{array}{l}\mathrm{Pd}(\mathrm{OAc})_{2}(1 \mathrm{~mol} \%), \\
1-10(2 \mathrm{~mol} \%), \mathrm{mw}(300 \mathrm{~W}) \\
\mathrm{DMF}^{2} \mathrm{H}_{2} \mathrm{O}(1: 1), 80^{\circ} \mathrm{C}, 10 \mathrm{~min} \\
\mathrm{~K}_{2} \mathrm{CO}_{3}(2 \text { equiv) }\end{array}$} & \multirow[b]{2}{*}{ Conversion $^{a}(\%)$} \\
\hline & & & $\mathbf{X}$ & Salt & \\
\hline 1 & $\mathrm{H}$ & $\mathrm{N}$ & $\mathrm{Br}$ & 1 & $67^{b}$ \\
\hline 2 & $\mathrm{H}$ & $\mathrm{N}$ & $\mathrm{Br}$ & 1 & $75^{\mathrm{c}}$ \\
\hline 3 & $\mathrm{H}$ & $\mathrm{N}$ & $\mathrm{Br}$ & 1 & $76^{d}$ \\
\hline 4 & $\mathrm{H}$ & $\mathrm{N}$ & $\mathrm{Br}$ & 1 & $06^{\mathrm{e}}$ \\
\hline 5 & $\mathrm{H}$ & $\mathrm{N}$ & $\mathrm{Br}$ & 1 & $11^{\mathrm{f}}$ \\
\hline 6 & $\mathrm{H}$ & $\mathrm{N}$ & $\mathrm{Br}$ & no & $32^{g}$ \\
\hline 7 & $\mathrm{H}$ & $\mathrm{CH}$ & I & 1 & 98 \\
\hline 8 & $\mathrm{H}$ & $\mathrm{CH}$ & I & 2 & 98 \\
\hline
\end{tabular}


Table 4. Cont.

\begin{tabular}{|c|c|c|c|c|c|}
\hline Entry & $\mathbf{R}$ & $\mathbf{Z}$ & $\mathbf{X}$ & Salt & Conversion $^{\mathrm{a}}(\%)$ \\
\hline 9 & $\mathrm{H}$ & $\mathrm{CH}$ & I & 3 & 97 \\
\hline 10 & $\mathrm{H}$ & $\mathrm{CH}$ & I & 4 & 97 \\
\hline 11 & $\mathrm{H}$ & $\mathrm{CH}$ & I & 5 & 96 \\
\hline 12 & $\mathrm{H}$ & $\mathrm{CH}$ & I & 6 & $9795^{\mathrm{i}}$ \\
\hline 13 & $\mathrm{H}$ & $\mathrm{CH}$ & I & 7 & $94^{\mathrm{i}}$ \\
\hline 14 & $\mathrm{H}$ & $\mathrm{CH}$ & I & 8 & $91^{\mathrm{i}}$ \\
\hline 15 & $\mathrm{H}$ & $\mathrm{CH}$ & I & 9 & $90^{\mathrm{i}}$ \\
\hline 16 & $\mathrm{H}$ & $\mathrm{CH}$ & I & 10 & $90^{\mathrm{i}}$ \\
\hline 17 & $\mathrm{OCH}_{3}$ & $\mathrm{CH}$ & $\mathrm{I}$ & 1 & 99 \\
\hline 18 & $\mathrm{OCH}_{3}$ & $\mathrm{CH}$ & I & 2 & 99 \\
\hline 19 & $\mathrm{OCH}_{3}$ & $\mathrm{CH}$ & I & 3 & 97 \\
\hline 20 & $\mathrm{OCH}_{3}$ & $\mathrm{CH}$ & I & 4 & 98 \\
\hline 21 & $\mathrm{OCH}_{3}$ & $\mathrm{CH}$ & I & 5 & 98 \\
\hline 22 & $\mathrm{OCH}_{3}$ & $\mathrm{CH}$ & I & 6 & $9795^{\mathrm{i}}$ \\
\hline 23 & $\mathrm{OCH}_{3}$ & $\mathrm{CH}$ & I & 7 & $95^{\mathrm{i}}$ \\
\hline 24 & $\mathrm{OCH}_{3}$ & $\mathrm{CH}$ & I & 8 & $94^{\mathrm{i}}$ \\
\hline 25 & $\mathrm{OCH}_{3}$ & $\mathrm{CH}$ & I & 9 & $94^{\mathrm{i}}$ \\
\hline 26 & $\mathrm{OCH}_{3}$ & $\mathrm{CH}$ & I & 10 & $93^{\mathrm{i}}$ \\
\hline 27 & $\mathrm{COCH}_{3}$ & $\mathrm{CH}$ & $\mathrm{Br}$ & 1 & 99 \\
\hline 28 & $\mathrm{COCH}_{3}$ & $\mathrm{CH}$ & $\mathrm{Br}$ & 2 & 99 \\
\hline 29 & $\mathrm{COCH}_{3}$ & $\mathrm{CH}$ & $\mathrm{Br}$ & 3 & 99 \\
\hline 30 & $\mathrm{COCH}_{3}$ & $\mathrm{CH}$ & $\mathrm{Br}$ & 4 & 99 \\
\hline 31 & $\mathrm{COCH}_{3}$ & $\mathrm{CH}$ & $\mathrm{Br}$ & 5 & 98 \\
\hline 32 & $\mathrm{COCH}_{3}$ & $\mathrm{CH}$ & $\mathrm{Br}$ & 6 & $9896^{\mathrm{i}}$ \\
\hline 33 & $\mathrm{COCH}_{3}$ & $\mathrm{CH}$ & $\mathrm{Br}$ & 7 & $96^{\mathrm{i}}$ \\
\hline 34 & $\mathrm{COCH}_{3}$ & $\mathrm{CH}$ & $\mathrm{Br}$ & 8 & $95^{\mathrm{i}}$ \\
\hline 35 & $\mathrm{COCH}_{3}$ & $\mathrm{CH}$ & $\mathrm{Br}$ & 9 & $95^{\mathrm{i}}$ \\
\hline 36 & $\mathrm{COCH}_{3}$ & $\mathrm{CH}$ & $\mathrm{Br}$ & 10 & $95^{\mathrm{i}}$ \\
\hline 37 & $\mathrm{H}$ & $\mathrm{N}$ & $\mathrm{Br}$ & 2 & 72 \\
\hline 38 & $\mathrm{H}$ & $\mathrm{N}$ & $\mathrm{Br}$ & 3 & 69 \\
\hline 39 & $\mathrm{H}$ & $\mathrm{N}$ & $\mathrm{Br}$ & 4 & 70 \\
\hline 40 & $\mathrm{H}$ & $\mathrm{N}$ & $\mathrm{Br}$ & 5 & 63 \\
\hline 41 & $\mathrm{H}$ & $\mathrm{N}$ & $\mathrm{Br}$ & 6 & 70 \\
\hline 42 & $\mathrm{H}$ & $\mathrm{N}$ & $\mathrm{Br}$ & 7 & 67 \\
\hline 43 & $\mathrm{H}$ & $\mathrm{N}$ & $\mathrm{Br}$ & 8 & 68 \\
\hline 44 & $\mathrm{H}$ & $\mathrm{N}$ & $\mathrm{Br}$ & 9 & 70 \\
\hline 45 & $\mathrm{H}$ & $\mathrm{N}$ & $\mathrm{Br}$ & 10 & 70 \\
\hline
\end{tabular}

${ }^{a}$ Conversions were determined by GC-MS based on the aryl halide. Reaction conditions: temperature ramped to $80^{\circ} \mathrm{C}(3 \mathrm{~min})$ and held for $5^{\mathrm{b}} \mathrm{min}, 10^{\mathrm{c}} \mathrm{min}$ and $20^{\mathrm{d}} \mathrm{min}$. In preheated oil bath, $5^{\mathrm{e}} \mathrm{min}$ and $10^{\mathrm{f}}$ min with thermal heating at $80^{\circ} \mathrm{C}$. Temperature ramped to $80^{\circ} \mathrm{C}(3 \mathrm{~min})$ and held for $10^{\mathrm{g}} \mathrm{min}$ without salt (1). Isolated yields ${ }^{\mathrm{i}}$.

The benzimidazole salts bearing an electron-withdrawing chloro substituent on the para-position of the phenyl ring, 6-10, were found to be the less effective of the salts examined in the Suzuki-Miyaura cross-coupling reactions (Table 4, entries 12-16, 22-26, 32-36 and 41-45). On the other hand, 
benzimidazole salts $\mathbf{1}$ and $\mathbf{6}$ bearing an electron-donating ethyl group on the nitrogen atom are the most effective for the catalytic activity in Suzuki coupling reactions. Similar catalytic results for the Suzuki-Miyaura cross-coupling reactions have also been obtained using $\mathrm{Pd}(\mathrm{OAc})_{2}$ or $\mathrm{PdCl}_{2}$, base and benzimidazole or imidazole catalytic systems bearing different electron-donating or electron-withdrawing aryl, substituted aryl, alkyl and substituted alkyl groups on benzimidazole or imidazole ligands [51-53]. A comparison of our catalytic system consisting of $\mathrm{Pd}(\mathrm{OAc})_{2} /$ benzimidazolium salt $/ \mathrm{K}_{2} \mathrm{CO}_{3}$ in $\mathrm{DMF} / \mathrm{H}_{2} \mathrm{O}$ under microwave heating with similar catalytic systems including NHC ligand [8,14,54-56], under conventional heating clearly indicates that microwave heating improves in catalytic yields and reaction times.

\section{Experimental}

\subsection{General Chemical Procedure}

Starting materials and reagents used were of commercial grade and purchased from Aldrich or Merck Chemical Co. Solvents were dried with standard methods and freshly distilled prior to use. All catalytic activity experiments were carried out in a microwave oven manufactured by Milestone (Milestone Start S Microwave Labstation for Synthesis, Sorisole, Italy) under aerobic conditions. ${ }^{1} \mathrm{H}-\mathrm{NMR}(300 \mathrm{MHz})$ and ${ }^{13} \mathrm{C}-\mathrm{NMR}(75 \mathrm{MHz})$ spectra were recorded using a Bruker DPX-300 high performance digital FT NMR spectrometer (Billercia, MA, USA). Infrared spectra were recorded from $\mathrm{KBr}$ pellets in the range $4000-400 \mathrm{~cm}^{-1}$ on a Perkin-Elmer FT-IR spectrophotometer. Elemental analyses were performed with a LECO CHNS-932 elemental analyzer (St. Joseph, MI, USA). Melting points were recorded using an electrothermal-9200 melting point apparatus, and are given uncorrected.

Synthesis of 3-ethyl-1-phenylbenzimidazolium iodide (1). To a solution of 1-phenylbenzimidazole $(1.35 \mathrm{~g}, 6.95 \mathrm{mmol})$ in dimethylformamide $(5 \mathrm{~mL})$ was added ethyl iodide $\left(0.60 \mathrm{~cm}^{3}, 6.95 \mathrm{mmol}\right)$ and the mixture was heated under reflux for $3 \mathrm{~h}$. The mixture was then cooled and the solvent was removed in vacuo. The residue was crystallized from $\mathrm{EtOH} / \mathrm{Et}_{2} \mathrm{O}$ (1:1). Yield $1.90 \mathrm{~g}, 78 \%$; m.p.: 203-204 ${ }^{\circ} \mathrm{C}$; $v_{(\mathrm{N}=\mathrm{C})}: 1560 \mathrm{~cm}^{-1}$. Anal. found: $\mathrm{C}, 51.32 ; \mathrm{H}, 4.29 ; \mathrm{N}, 7.94 \%$. Calcd for $\mathrm{C}_{15} \mathrm{H}_{15} \mathrm{~N}_{2} \mathrm{I}: \mathrm{C}, 51.45 ; \mathrm{H}, 4.32 ; \mathrm{N}$, 8.00\%. ${ }^{1} \mathrm{H}-\mathrm{NMR}\left(300.13 \mathrm{MHz}, \mathrm{DMSO}-\mathrm{d}_{6}\right): \delta 10.20(\mathrm{~s}, 1 \mathrm{H}, \mathrm{NCHN}), 8.24\left(\mathrm{~d}, 1 \mathrm{H}, \mathrm{C}_{6} \mathrm{H}_{4}, J=7.5 \mathrm{~Hz}\right)$, $7.86\left(\mathrm{~m}, 3 \mathrm{H}, \mathrm{C}_{6} \mathrm{H}_{4}\right), 7.78-7.70\left(\mathrm{~m}, 5 \mathrm{H}, \mathrm{C}_{6} \mathrm{H}_{5}\right), 4.63\left(\mathrm{q}, 2 \mathrm{H}, \mathrm{CH}_{2} \mathrm{CH}_{3}, J=7.2 \mathrm{~Hz}\right), 1.64\left(\mathrm{t}, 3 \mathrm{H}, \mathrm{CH}_{2} \mathrm{CH}_{3}\right.$, $J=7.2 \mathrm{~Hz}) .{ }^{13} \mathrm{C}-\mathrm{NMR}\left(75.47 \mathrm{MHz}, \mathrm{DMSO}-\mathrm{d}_{6}\right): \delta 142.8(\mathrm{NCHN}), 133.7,131.6,131.5,130.9,130.8$, 127.9, 127.4, 125.7, 114.5, $113.9\left(\mathrm{C}_{6} \mathrm{H}_{4}\right.$ and $\left.\mathrm{C}_{6} \mathrm{H}_{5}\right), 42.9\left(\mathrm{CH}_{2} \mathrm{CH}_{3}\right), 14.5\left(\mathrm{CH}_{2} \mathrm{CH}_{3}\right)$.

Synthesis of 3-(3-cyanopropyl)-1-phenylbenzimidazolium chloride (2). This compound was similarly was synthesized from 1-phenylbenzimidazole and 4-chloro-butyronitrile. Yield $1.54 \mathrm{~g}$, 74\%; m.p.: 101-102 ${ }^{\circ} \mathrm{C} ; \mathrm{v}_{(\mathrm{N}=\mathrm{C})}: 1557 \mathrm{~cm}^{-1}$. Anal. found: $\mathrm{C}, 68.44 ; \mathrm{H}, 5.35 ; \mathrm{N}, 14.06 \%$. Calcd for $\mathrm{C}_{17} \mathrm{H}_{16} \mathrm{~N}_{3} \mathrm{Cl}: \mathrm{C}$, 68.57; H, 5.42; N, 14.11\%. ${ }^{1} \mathrm{H}-\mathrm{NMR}\left(300.13 \mathrm{MHz}, \mathrm{DMSO}-\mathrm{d}_{6}\right): \delta 10.74$ (s, 1H, NCHN), 8.35 (d, 1H, $\left.\mathrm{C}_{6} \mathrm{H}_{4}, J=8.1 \mathrm{~Hz}\right), 7.91-7.84\left(\mathrm{~m}, 3 \mathrm{H}, \mathrm{C}_{6} \mathrm{H}_{4}\right), 7.79-7.68\left(\mathrm{~m}, 5 \mathrm{H}, \mathrm{C}_{6} \mathrm{H}_{5}\right), 4.81\left(\mathrm{t}, 2 \mathrm{H}, \mathrm{CH}_{2} \mathrm{CH}_{2} \mathrm{CH}_{2} \mathrm{CN}\right.$, $J=6.6 \mathrm{~Hz}), 2.86\left(\mathrm{t}, 2 \mathrm{H}, \mathrm{CH}_{2} \mathrm{CH}_{2} \mathrm{CH}_{2} \mathrm{CN}, J=7.2 \mathrm{~Hz}\right), 2.40\left(\mathrm{~m}, 2 \mathrm{H}, \mathrm{CH}_{2} \mathrm{CH}_{2} \mathrm{CH}_{2} \mathrm{CN}\right) .{ }^{13} \mathrm{C}-\mathrm{NMR}$ (75.47 MHz, DMSO-d 6 ): $\delta 143.6(\mathrm{NCHN}), 133.7,131.7,131.4,130.8,127.9,127.4,125.6,120.5,114.6$, $113.9\left(\mathrm{C}_{6} \mathrm{H}_{4}\right.$ and $\left.\mathrm{C}_{6} \mathrm{H}_{5}\right), 46.3\left(\mathrm{CH}_{2} \mathrm{CH}_{2} \mathrm{CH}_{2} \mathrm{CN}\right), 34.3\left(\mathrm{CH}_{2} \mathrm{CH}_{2} \mathrm{CH}_{2} \mathrm{CN}\right), 25.0\left(\mathrm{CH}_{2} \mathrm{CH}_{2} \mathrm{CH}_{2} \mathrm{CN}\right), 14.3$ $\left(\mathrm{CH}_{2} \mathrm{CH}_{2} \mathrm{CH}_{2} \mathrm{CN}\right)$. 
Synthesis of 3-(2-vinyloxy)ethyl-1-phenylbenzimidazolium chloride (3). Synthesized from 1-phenylbenzimidazole and 2-chloroethyl vinyl ether. Yield 1.66 g, 79\%; m.p.: $112-113{ }^{\circ} \mathrm{C}$; v (N=C): $1558 \mathrm{~cm}^{-1}$. Anal. found: C, 67.76; H, 5.64; N, 9.22\%. Calcd for $\mathrm{C}_{17} \mathrm{H}_{17} \mathrm{~N}_{2} \mathrm{OCl}$ : C, 67.88; H, 5.70; N, 9.31\%. ${ }^{1} \mathrm{H}-\mathrm{NMR}\left(300.13 \mathrm{MHz}, \mathrm{DMSO}-\mathrm{d}_{6}\right): \delta 10.32(\mathrm{~s}, 1 \mathrm{H}, \mathrm{NCHN}), 8.25\left(\mathrm{~d}, 1 \mathrm{H}, \mathrm{C}_{6} \mathrm{H}_{4}, J=7.8 \mathrm{~Hz}\right), 7.90-7.87$ (m, 3H, C6H4), 7.80-7.70 (m, 5H, $\left.\mathrm{C}_{6} \mathrm{H}_{5}\right), 5.52\left(\mathrm{t}, 1 \mathrm{H}, \mathrm{CH}_{2} \mathrm{CH}_{2} \mathrm{OCH}=\mathrm{CH}_{2}, J=5.7 \mathrm{~Hz}\right), 4.69$ (t, 2H, $\mathrm{CH}_{2} \mathrm{CH}_{2} \mathrm{OCH}=\mathrm{CH}_{2}, J=4.6 \mathrm{~Hz}$ ), 3.92 (two d, $2 \mathrm{H}, \mathrm{CH}_{2} \mathrm{CH}_{2} \mathrm{OCH}=\mathrm{CHH}, J=4.8 \mathrm{~Hz}$ and $15 \mathrm{~Hz}$ ), 3.40 (t, $\left.2 \mathrm{H}, \mathrm{CH}_{2} \mathrm{CH}_{2} \mathrm{OCH}=\mathrm{CH}_{2}, J=4.6 \mathrm{~Hz}\right) .{ }^{13} \mathrm{C}-\mathrm{NMR}\left(75.47 \mathrm{MHz}, \mathrm{DMSO}-\mathrm{d}_{6}\right): \delta 143.3(\mathrm{NCHN}), 143.4$, 133.7, 132.1, 131.5, 130.9, 130.8, 127.7, 127.2, $125.7\left(\mathrm{C}_{6} \mathrm{H}_{4}\right.$ and $\left.\mathrm{C}_{6} \mathrm{H}_{5}\right), 114.9,113.8\left(\mathrm{CH}=\mathrm{CH}_{2}\right), 59.2$ $\left(\mathrm{CH}_{2} \mathrm{CH}_{2} \mathrm{OCH}=\mathrm{CH}_{2}\right), 50.4\left(\mathrm{CH}_{2} \mathrm{CH}_{2} \mathrm{OCH}=\mathrm{CH}_{2}\right)$.

Synthesis of 3-allyl-1-phenylbenzimidazolium bromide (4). Synthesized from 1-phenylbenzimidazole and allyl bromide. Yield 1.87 g, 85\%; m.p.: $171-172{ }^{\circ} \mathrm{C}$; $v_{(\mathrm{N}=\mathrm{C})}: 1554 \mathrm{~cm}^{-1}$. Anal. found: $\mathrm{C}, 60.91 ; \mathrm{H}$, 4.73; N, 8.73\%. Calcd for $\mathrm{C}_{16} \mathrm{H}_{15} \mathrm{~N}_{2} \mathrm{Br}$ : C, 60.97; H, 4.80; N, 8.89\%. ${ }^{1} \mathrm{H}-\mathrm{NMR}$ (300.13 MHz, DMSO-d 6 ): $\delta 10.28(\mathrm{~s}, 1 \mathrm{H}, \mathrm{NCHN}), 8.40\left(\mathrm{~d}, 1 \mathrm{H}, \mathrm{C}_{6} \mathrm{H}_{4}, J=7.2 \mathrm{~Hz}\right), 7.96-7.85(\mathrm{~m}, 3 \mathrm{H}, \mathrm{C} 6 \mathrm{H} 4,7.82-7.77$ (m, 5H, $\left.\mathrm{C}_{6} \mathrm{H}_{5}\right), 6.26-6.13\left(\mathrm{~m}, 1 \mathrm{H}, \mathrm{CH}_{2} \mathrm{CH}=\mathrm{CH}_{2}\right), 5.60\left(\mathrm{dd}, 1 \mathrm{H}, \mathrm{CH}_{2} \mathrm{CH}=\mathrm{CHH}, J=15.9 ; 1.2 \mathrm{~Hz}\right), 5.46(\mathrm{dd}, 1 \mathrm{H}$, $\left.\mathrm{CH}_{2} \mathrm{CH}=\mathrm{CHH}, J=9.0 ; 1.2 \mathrm{~Hz}\right), 5.30\left(\mathrm{~d}, 2 \mathrm{H}, \mathrm{CH}_{2} \mathrm{CH}=\mathrm{CH}_{2}, J=6.0 \mathrm{~Hz}\right) .{ }^{13} \mathrm{C}-\mathrm{NMR}(75.47 \mathrm{MHz}$, DMSO-d $\left.{ }_{6}\right): \delta 143.3(\mathrm{NCHN}), 133.6,131.7,131.5,131.2,130.9,130.8,127.9,127.4,125.7,121.4$ $\left(\mathrm{C}_{6} \mathrm{H}_{4}\right.$ and $\left.\mathrm{C}_{6} \mathrm{H}_{5}\right), 114.7,114.1\left(\mathrm{CH}_{2} \mathrm{CH}=\mathrm{CH}_{2}\right), 49.7\left(\mathrm{CH}_{2} \mathrm{CH}=\mathrm{CH}_{2}\right)$.

Synthesis of 3-p-chlorobenzyl-1-phenylbenzimidazolium chloride (5). Synthesized from 1-phenylbenzimidazole and p-chlorobenzyl chloride. Yield $1.88 \mathrm{~g}, 76 \%$; m.p.: $82-83{ }^{\circ} \mathrm{C}$; $v_{(\mathrm{N}=\mathrm{C})}: 1558 \mathrm{~cm}^{-1}$. Anal. found: C, 67.45; H, 4.51; N, 7.73\%. Calcd for $\mathrm{C}_{20} \mathrm{H}_{16} \mathrm{~N}_{2} \mathrm{Cl}_{2}: \mathrm{C}, 67.62 ; \mathrm{H}, 4.54 ; \mathrm{N}, 7.89 \%$. ${ }^{1} \mathrm{H}-\mathrm{NMR}$ (300.13 MHz, DMSO-d $\left.)_{6}\right): \delta 10.51(\mathrm{~s}, 1 \mathrm{H}, \mathrm{NCHN}), 8.06-8.03\left(\mathrm{~m}, 1 \mathrm{H}, \mathrm{C}_{6} \mathrm{H}_{4}\right), 7.91-7.86\left(\mathrm{~m}, 3 \mathrm{H}, \mathrm{C}_{6} \mathrm{H}_{4}\right)$, 7.76-7.73 (m, 5H, $\left.\mathrm{C}_{6} \mathrm{H}_{5}\right), 7.71\left(\mathrm{~d}, 2 \mathrm{H}, \mathrm{C}_{6} \mathrm{H}_{4}, J=8.4 \mathrm{~Hz}\right), 5.89\left(\mathrm{~s}, 2 \mathrm{H}, \mathrm{CH}_{2}\right) .{ }^{13} \mathrm{C}-\mathrm{NMR}(75.47 \mathrm{MHz}$, DMSO-d $\left.{ }_{6}\right): \delta 143.6(\mathrm{NCHN}), 134.0,133.6,133.1,131.8,131.3,131.0,130.8,129.4,128.0,127.6$, 125.8, 114.6, $114.2\left(\mathrm{C}_{6} \mathrm{H}_{4}\right.$ and $\left.\mathrm{C}_{6} \mathrm{H}_{5}\right), 49.9\left(\mathrm{NCH}_{2}-\right)$.

Synthesis of 3-Ethyl-1-p-chlorophenylbenzimidazolium iodide (6). To a solution of 1-p-chlorophenylbenzimidazole $(1.37 \mathrm{~g}, 6.0 \mathrm{mmol})$ in dimethylformamide $(5 \mathrm{~mL})$ was added ethyl iodide $\left(0.50 \mathrm{~cm}^{3}, 6.0 \mathrm{mmol}\right)$ and the mixture was heated under reflux for $3 \mathrm{~h}$. The mixture was then cooled and the solvent was removed in vacuo. The residue was crystallized from $\mathrm{EtOH} / \mathrm{Et}_{2} \mathrm{O}(1: 1)$. Yield $1.98 \mathrm{~g}, 86 \%$; m.p.: 216-217 ${ }^{\circ} \mathrm{C}$; $v_{(\mathrm{N}=\mathrm{C})}: 1559 \mathrm{~cm}^{-1}$. Anal. found: C, 46.56; H, 3.63; N, 7.09\%. Calcd for $\mathrm{C}_{15} \mathrm{H}_{14} \mathrm{~N}_{2} \mathrm{ICl}: \mathrm{C}, 46.84 ; \mathrm{H}, 3.67 ; \mathrm{N}$, 7.28\%. ${ }^{1} \mathrm{H}-\mathrm{NMR}$ (300.13 MHz, DMSO-d 6 ): $\delta 10.18$ (s, 1H, NCHN), $8.23\left(\mathrm{~d}, 1 \mathrm{H}, \mathrm{C}_{6} \mathrm{H}_{4}, J=6.3 \mathrm{~Hz}\right.$ ), $7.92-7.71\left(\mathrm{~m}, 7 \mathrm{H}, \mathrm{C}_{6} \mathrm{H}_{4}\right.$ and $\mathrm{C}_{6} \mathrm{H}_{4} \mathrm{Cl}$ ), 4.62 (q, 2H, $\left.\mathrm{CH}_{2} \mathrm{CH}_{3}, J=7.3 \mathrm{~Hz}\right), 1.63$ (t, 3H, $\mathrm{CH}_{2} \mathrm{CH}_{3}, J=7.3 \mathrm{~Hz}$ ). ${ }^{13} \mathrm{C}-\mathrm{NMR}\left(75.47 \mathrm{MHz}, \mathrm{DMSO}-\mathrm{d}_{6}\right): \delta 142.9(\mathrm{NCHN}), 133.5,132.6,131.6,131.4,130.8,127.9,127.7$, 127.4, 114.5, $113.9\left(\mathrm{C}_{6} \mathrm{H}_{4}\right.$ and $\left.\mathrm{C}_{6} \mathrm{H}_{4} \mathrm{Cl}\right), 42.9\left(\mathrm{CH}_{2} \mathrm{CH}_{3}\right), 14.4\left(\mathrm{CH}_{2} \mathrm{CH}_{3}\right)$.

Synthesis of 3-(3-cyanopropyl)-1-p-chlorophenylbenzimidazolium chloride (7). Similarly synthesized from 1-p-chlorophenylbenzimidazole and 4-chlorobutyronitrile. Yield 1.44 g, 72\%; m.p.: 101-102 ${ }^{\circ} \mathrm{C}$; $v_{(\mathrm{N}=\mathrm{C})}: 1557 \mathrm{~cm}^{-1}$. Anal. found: $\mathrm{C}, 61.14 ; \mathrm{H}, 4.43 ; \mathrm{N}, 12.18 \%$. Calcd for $\mathrm{C}_{17} \mathrm{H}_{15} \mathrm{~N}_{3} \mathrm{Cl}_{2}: \mathrm{C}, 61.46 ; \mathrm{H}$, 4.55; N, 12.65\%. ${ }^{1} \mathrm{H}-\mathrm{NMR}\left(300.13 \mathrm{MHz}, \mathrm{DMSO}-\mathrm{d}_{6}\right): \delta 10.51(\mathrm{~s}, 1 \mathrm{H}, \mathrm{NCHN}), 8.27\left(\mathrm{~d}, 1 \mathrm{H}, \mathrm{C}_{6} \mathrm{H}_{4}\right.$, $J=8.1 \mathrm{~Hz}), 7.93-7.71\left(\mathrm{~m}, 7 \mathrm{H}, \mathrm{C}_{6} \mathrm{H}_{4}\right.$ and $\left.\mathrm{C}_{6} \mathrm{H}_{4} \mathrm{Cl}\right), 4.72\left(\mathrm{t}, 2 \mathrm{H}, \mathrm{CH}_{2} \mathrm{CH}_{2} \mathrm{CH}_{2} \mathrm{CN}, J=6.45 \mathrm{~Hz}\right), 2.81$ (t, $2 \mathrm{H}, \mathrm{CH}_{2} \mathrm{CH}_{2} \mathrm{CH}_{2} \mathrm{CN}, J=7.2 \mathrm{~Hz}$ ), 2.38 (m, $2 \mathrm{H}, \mathrm{CH}_{2} \mathrm{CH}_{2} \mathrm{CH}_{2} \mathrm{CN}$ ). ${ }^{13} \mathrm{C}-\mathrm{NMR}\left(75.47 \mathrm{MHz}, \mathrm{DMSO}-\mathrm{d}_{6}\right.$ ): 
$\delta 143.7(\mathrm{NCHN}), 135.5,132.5,131.6,131.5,130.9,127.9,127.7,120.4,114.5,113.9\left(\mathrm{C}_{6} \mathrm{H}_{4}\right.$ and $\left.\mathrm{C}_{6} \mathrm{H}_{4} \mathrm{Cl}\right)$, $46.3\left(\mathrm{CH}_{2} \mathrm{CH}_{2} \mathrm{CH}_{2} \mathrm{CN}\right), 34.4\left(\mathrm{CH}_{2} \mathrm{CH}_{2} \mathrm{CH}_{2} \mathrm{CN}\right), 24.9\left(\mathrm{CH}_{2} \mathrm{CH}_{2} \mathrm{CH}_{2} \mathrm{CN}\right), 14.3\left(\mathrm{CH}_{2} \mathrm{CH}_{2} \mathrm{CH}_{2} \mathrm{CN}\right)$.

Synthesis of 3-(2-vinyloxyethyl)-1-p-chlorophenylbenzimidazolium chloride (8). Synthesized from 1-pchlorophenylbenzimidazole and 2-chloroethyl vinyl ether. Yield 1.41 g, 70\%; m.p.: $164-166{ }^{\circ} \mathrm{C}$; v $(\mathrm{N}=\mathrm{C}): 1557 \mathrm{~cm}^{-1}$. Anal. found: $\mathrm{C}, 60.26 ; \mathrm{H}, 4.64 ; \mathrm{N}, 8.08 \%$. Calcd for $\mathrm{C}_{17} \mathrm{H}_{16} \mathrm{~N}_{2} \mathrm{OCl}_{2}$ : C, 60.91; $\mathrm{H}$, 4.81; N, 8.36\%. ${ }^{1} \mathrm{H}-\mathrm{NMR}\left(300.13 \mathrm{MHz}, \mathrm{DMSO}_{-} \mathrm{d}_{6}\right): \delta 10.30(\mathrm{~s}, 1 \mathrm{H}, \mathrm{NCHN}), 8.25\left(\mathrm{~d}, 1 \mathrm{H}, \mathrm{C}_{6} \mathrm{H}_{4}\right.$, $J=8.4 \mathrm{~Hz}$ ), 7.94-7.70 (m, 7H, $\mathrm{C}_{6} \mathrm{H}_{4}$ and $\left.\mathrm{C}_{6} \mathrm{H}_{4} \mathrm{Cl}\right), 5.54\left(\mathrm{t}, 1 \mathrm{H}, \mathrm{CH}_{2} \mathrm{CH}_{2} \mathrm{OCH}=\mathrm{CH}_{2}, J=6.0 \mathrm{~Hz}\right), 4.68$ $\left(\mathrm{t}, 2 \mathrm{H}, \mathrm{CH}_{2} \mathrm{CH}_{2} \mathrm{OCH}=\mathrm{CH}_{2}, J=4.6 \mathrm{~Hz}\right), 3.92\left(\mathrm{dd}, 2 \mathrm{H}, \mathrm{CH}_{2} \mathrm{CH}_{2} \mathrm{OCH}=\mathrm{CHH}, J=5.0 \mathrm{~Hz}\right), 3.40(\mathrm{t}$, $\mathrm{CH}_{2} \mathrm{CH}_{2} \mathrm{CH}_{2} \mathrm{CN}$ ). ${ }^{13} \mathrm{C}-\mathrm{NMR}\left(75.47 \mathrm{MHz}, \mathrm{DMSO}-\mathrm{d}_{6}\right): \delta 143.5$ (NCHN), 133.5, 131.9, 131.6, 130.8, 127.8, 127.7, $127.3\left(\mathrm{C}_{6} \mathrm{H}_{4}\right.$ and $\left.\mathrm{C}_{6} \mathrm{H}_{4} \mathrm{Cl}\right), 114.8,113.8\left(\mathrm{CH}=\mathrm{CH}_{2}\right), 59.1\left(\mathrm{CH}_{2} \mathrm{CH}_{2} \mathrm{OCH}=\mathrm{CH}_{2}\right), 50.5$ $\left(\mathrm{CH}_{2} \mathrm{CH}_{2} \mathrm{OCH}=\mathrm{CH}_{2}\right)$.

Synthesis of 3-Allyl-1-p-chlorophenylbenzimidazolium Bromide (9). Synthesized from 1-p-chlorophenylbenzimidazole and allyl bromide. Yield $1.93 \mathrm{~g}, 92 \%$; m.p.: $119-121{ }^{\circ} \mathrm{C}$; $v_{(\mathrm{N}=\mathrm{C})}: 1552 \mathrm{~cm}^{-1}$. Anal. found: C, 54.23; $\mathrm{H}, 4.03 ; \mathrm{N}, 7.83 \%$. Calcd for $\mathrm{C}_{16} \mathrm{H}_{14} \mathrm{~N}_{2} \mathrm{BrCl}$ : C, 54.96; H, 4.04; N, 8.01\%. ${ }^{1} \mathrm{H}-\mathrm{NMR}$ (300.13 MHz, DMSO-d ${ }_{6}$ ): $\delta 10.34(\mathrm{~s}, 1 \mathrm{H}, \mathrm{NCHN}), 8.14\left(\mathrm{~d}, 1 \mathrm{H}, \mathrm{C}_{6} \mathrm{H}_{4}, J=8.4 \mathrm{~Hz}\right), 7.94-7.70(\mathrm{~m}, 7 \mathrm{H}$, $\mathrm{C}_{6} \mathrm{H}_{4}$ and $\left.\mathrm{C}_{6} \mathrm{H}_{4} \mathrm{Cl}\right), 6.26-6.14\left(\mathrm{~m}, 1 \mathrm{H}, \mathrm{CH}_{2} \mathrm{CH}=\mathrm{CH}_{2}\right), 5.60(\mathrm{~d}, 1 \mathrm{H}, \mathrm{CH}=\mathrm{CHH}, J=17.1 \mathrm{~Hz}), 5.46(\mathrm{~d}$, $\left.1 \mathrm{H}, \mathrm{CH}_{2} \mathrm{CH}=\mathrm{CHH}, J=10.2 \mathrm{~Hz}\right), 5.32\left(\mathrm{~d}, 2 \mathrm{H}, \mathrm{CH}_{2} \mathrm{CH}=\mathrm{CH}_{2}, J=5.7 \mathrm{~Hz}\right) .{ }^{13} \mathrm{C}-\mathrm{NMR}(75.47 \mathrm{MHz}$, DMSO-d $\left.)_{6}\right): \delta 143.4(\mathrm{NCHN}), 135.5,132.5,131.7,131.4,131.1,130.8,127.9,127.8,127.4,121.4\left(\mathrm{C}_{6} \mathrm{H}_{4}\right.$ and $\left.\mathrm{C}_{6} \mathrm{H}_{4} \mathrm{Cl}\right), 114.7,114.0\left(\mathrm{CH}_{2} \mathrm{CH}=\mathrm{CH}_{2}\right), 49.7\left(\mathrm{CH}_{2} \mathrm{CH}=\mathrm{CH}_{2}\right)$.

Synthesis of 3-p-Chlorobenzyl-1-p-chlorophenylbenzimidazolium Chloride (10). Synthesized from 1-pchlorophenylbenzimidazole and p-chlorobenzyl chloride. Yield 2.01 g, 86\%; m.p.: 209-210 ${ }^{\circ} \mathrm{C} ; v_{(\mathrm{N}=\mathrm{C})}$ : $1554 \mathrm{~cm}^{-1}$. Anal. found: C, 61.05; H, 3.79; N, 7.02\%. Calcd for $\mathrm{C}_{20} \mathrm{H}_{15} \mathrm{~N}_{2} \mathrm{Cl}_{3}: \mathrm{C}, 61.64 ; \mathrm{H}, 3.88 ; \mathrm{N}$, 7.19\%. ${ }^{1} \mathrm{H}-\mathrm{NMR}\left(300.13 \mathrm{MHz}, \mathrm{DMSO}-\mathrm{d}_{6}\right): \delta 10.67(\mathrm{~s}, 1 \mathrm{H}, \mathrm{NCHN}), 8.06-7.49\left(\mathrm{~m}, 12 \mathrm{H}, \mathrm{C}_{6} \mathrm{H}_{4}, \mathrm{C}_{6} \mathrm{H}_{4} \mathrm{Cl}\right.$ and $\left.\mathrm{CH}_{2} \mathrm{C}_{6} \mathrm{H}_{4} \mathrm{Cl}\right), 5.92\left(\mathrm{~s}, 2 \mathrm{H}, \mathrm{CH}_{2}\right) \cdot{ }^{13} \mathrm{C}-\mathrm{NMR}\left(75.47 \mathrm{MHz}, \mathrm{DMSO}-\mathrm{d}_{6}\right): \delta 143.7(\mathrm{NCHN}), 135.5$, $134.0,133.5,133.1,132.5,131.8,131.1,130.7,129.3,128.0,127.7,127.5,114.6,114.1\left(\mathrm{C}_{6} \mathrm{H}_{4}\right.$, $\mathrm{NC}_{6} \mathrm{H}_{4} \mathrm{Cl}$ and $\left.\mathrm{NCH}_{2} \mathrm{C}_{6} \mathrm{H}_{4} \mathrm{Cl}\right), 49.9\left(\mathrm{NCH}_{2}\right)$.

\subsection{General Procedure for the Heck-Mizoroki Reactions}

$\operatorname{Pd}(\mathrm{OAc})_{2}(1 \mathrm{mmol} \%)$, benzimidazolium halides 1-10 $(2 \mathrm{mmol} \%)$, the aryl halide (1 mmol), styrene $(1.2 \mathrm{mmol}), \mathrm{K}_{2} \mathrm{CO}_{3}(2 \mathrm{mmol})$, water $(3 \mathrm{~mL})$, and DMF $(3 \mathrm{~mL})$ were added to a microwave apparatus and the mixture was heated at $80{ }^{\circ} \mathrm{C}(300 \mathrm{~W})$ for $10 \mathrm{~min}$. A ramp time of 3 min was used to reach the temperature of $80{ }^{\circ} \mathrm{C}$. At the end of reaction, the mixture was cooled; the product was extracted with ethyl acetate/ $n$-hexane (1:5) and filtered through a pad of silica gel with copious washing. The percent conversion was determined by GC-MS based on aryl halide using the normalized peak areas method. The Heck-Mizoroki coupling yields between styrene with phenyl iodide, 4-iodoanisole or 4-bromo-acetophenone were also determined as an isolated yield for the comparison purposes with the GC based conversion (Table 2, entries 12-16, 22-26 and 32-36). The isolated yields were determined as follow: at the end of the coupling reaction, the mixture was cooled to room temperature; the contents of the reaction vessel were poured into a separatory funnel. Water $(3 \mathrm{~mL})$ 
and ethyl acetate $(5 \mathrm{~mL})$ were added, and the coupling product was extracted and removed. After further extraction of the aqueous phase with ethyl acetate $(5 \mathrm{~mL})$ and combining the extracts, the ethyl acetate was removed in vacuo leaving the trans-stilbene or corresponding derivatives which was characterized by comparison of NMR data with that in the literature.

\subsection{General Procedure for the Suzuki Reactions}

$\mathrm{Pd}(\mathrm{OAc})_{2}(1 \mathrm{mmol} \%)$, benzimidazolium halides 1-10 (2 mmol \%), aryl halide (1 mmol), phenylboronic acid $(1.2 \mathrm{mmol}), \mathrm{K}_{2} \mathrm{CO}_{3}(2 \mathrm{mmol})$, water $(3 \mathrm{~mL})$, DMF $(3 \mathrm{~mL})$ were added to microwave apparatus and the mixture was heated at $80{ }^{\circ} \mathrm{C}(300 \mathrm{~W})$ for $10 \mathrm{~min}$. A ramp time of $3 \mathrm{~min}$ was used to reach the temperature of $80^{\circ} \mathrm{C}$. At the end of reaction, the mixture was cooled, the product extracted with ethyl acetate/ $n$-hexane (1:5), chromatographed on a silica gel column. The purity of coupling products was checked by NMR and GC-MS, and yields are based on aryl halide. The coupling products were confirmed by increasing the peaks on gas chromatograms and mass values from MS spectrums. All coupling products were also isolated and characterized by ${ }^{1} \mathrm{H}-\mathrm{NMR}$ or MS before the serial catalytic work up each time. The Suzuki coupling yields between phenylboronic acid and phenyl iodide, 4-iodoanisole or 4-bromoacetophenone were also determined as an isolated yield for comparison purposes with the GC based yields (Table 4 entries, 12-16, 22-26 and 32-36). The isolated yields were determined as follows: at the end of the Suzuki coupling reaction, the mixture was cooled to room temperature, the contents of the reaction vessel were poured into a separatory funnel. Water $(3 \mathrm{~mL})$ and ethyl acetate $(5 \mathrm{~mL})$ were added, and the coupling product was extracted and removed. After further extraction of the aqueous phase with ethyl acetate $(5 \mathrm{~mL})$ and combining the extracts, the ethyl acetate was removed in vacuo leaving the coupling product which was characterized by comparison of NMR data with that in the literature.

\section{Conclusions}

We have prepared ten non-symmetric 1,3-disubstituted benzimidazolium salts 1-10 bearing on the nitrogen atoms of azolium ring phenyl, 4-chlorophenyl, 3-cyanopropyl, 2-vinyloxyethyl, allyl and 4-chlorobenzyl substituents. The catalytic activity of the novel benzimidazolium salts were evaluated using catalytic systems consisting of $\mathrm{Pd}(\mathrm{OAc})_{2}$ /benzimidazolium salt and $\mathrm{K}_{2} \mathrm{CO}_{3}$ for the HeckMizoroki and Suzuki-Miyaura cross coupling reactions. The catalyst systems in the Heck-Mizoroki and Suzuki-Miyaura reactions gave better yields under microwave-assisted moderate conditions after very short reaction times compared to those given in the literature [52,57-59]. In addition, coupling of 2-pyridyl bromide with both styrene and phenylboronic acid afford the corresponding stilbene and biaryl products in satisfactory yields after $10 \mathrm{~min}$.

\section{Acknowledgments}

This work was financially supported by the İnönü University Research Fund (I.Ü. B.A.P. 2011/144). 


\section{Supplementary Materials}

NMR spectra of the new compounds and some coupling products are available free of charge via the internet at http://www.mdpi.com/1420-3049/18/3/2501/s1.

\section{References}

1. Firouzabadi, H.; Iranpoor, N.; Kaazemi, F. Carbon-carbon bond formation via homocoupling reaction of substrates with a broad diversity in water using $\mathrm{Pd}(\mathrm{OAc})_{2}$ and agarose hydrogel as a bioorganic ligand, support and reductant. J. Mol. Cat. A: Chem. 2011, 348, 94-99.

2. Miyaura, N.; Suzuki, A. Palladium-Catalyzed Cross-Coupling Reactions of Organoboron Compounds. Chem. Rev. 2009, 95, 2457-2483.

3. Suzuki, A. Carbon-carbon bonding made easy. Chem. Commun. 2005, 4759-4763.

4. Ananikov, V.P.; Beletskaya, I.P. Toward the ideal catalyst: From atomic centers to a "Cocktail" of catalysts. Organometallics 2012, 31, 1595-1604.

5. Alonso, F.; Beletskaya, I.P.; Yus, M. Non-conventional methodologies for transition-metal catalysed carbon-carbon coupling: A critical overview. Part 2: The Suzuki reaction. Tetrahedron 2008, 64, 3047-3101.

6. Beletskaya, I.P.; Cheprakov, A.V. The Heck reaction as a sharpening stone of palladium catalysis. Chem. Rev. 2000, 100, 3009-3066.

7. Molnár, Á. Efficient, selective, and recyclable palladium catalysts in carbon-carbon coupling reactions. Chem. Rev. 2011, 111, 2251-2320.

8. Dawood, K.M.; Fayed, M.S.; Elkhalea, M.M. Heck and Suzuki cross-couplings of aryl and heteroaryl bromides in water using a new palladium(II)-complex. ARKIVOC 2009, xiii, 324-341.

9. Alonso, D.A.; Najera, C. Oxime-derived palladacycles as source of palladium nanoparticles. Chem. Soc. Rev. 2010, 39, 2891-2902.

10. Li, C.-J.; Chen, L. Organic chemistry in water. Chem. Rev. 2006, 35, 68-82.

11. Dallinger, D.; Kappe, C.O. Microwave-assisted synthesis in water as solvent. Chem. Rev. 2007, 107, 2563-2591.

12. Chanda, A.; Fokin, V.V. Organic synthesis "On Water". Chem. Rev. 2009, 109, 725-748.

13. Liu, L.-J.; Wang, F.; Shi, M. Elimination of an alkyl group from imidazolium salts: ImidazoleCoordinated dinuclear monodentate NHC-Palladium complexes driven by self-assembly and their application in the Heck reaction. Eur. J. Inorg. Chem. 2009, 1723-1728.

14. Kantchev, E.A.B.; O’Brien, C.J.; Organ, M.G. Palladium complexes of $N$-heterocyclic carbenes as catalysts for cross-coupling reactions-A synthetic chemist's perspective. Angew. Chem. Int. Ed. 2007, 46, 2768-2813.

15. Linninger, C.S.; Herdtweck, E.; Hoffmann, S.D.; Herrmann, W.A. A new palladium(II) complex of a functionalized $N$-heterocyclic carbene: Synthesis, characterization and application in Suzuki-miyaura cross-coupling reactions. J. Mol. Struct. 2008, 890, 192-197.

16. Marion, N.; Nolan, S.P. Well-defined $N$-heterocyclic Carbenes-Palladium(II) precatalysts for cross-coupling reactions. Account. Chem. Res. 2008, 41, 1440-1449. 
17. Mercan, D.; Çetinkaya, E.; Çetinkaya, B. Influence of $\mathrm{CH}_{3}$ substituents on tetrahydropyrimidin-2ylidene: Sigma-donating properties and in situ catalytic activities of precursor salts $/ \mathrm{Pd}(\mathrm{OAc})_{2}$ system for C-C coupling reactions. J. Organomet. Chem. 2011, 696, 1359-1366.

18. Korolev, D.N.; Bumagin, N.A. An improved protocol for ligandless Suzuki-Miyaura coupling in water. Tetrahedron Lett. 2006, 47, 4225-4229.

19. Tang, Y.-Q.; Chu, C.-Y.; Zhu, L.; Qian, B.; Shao, L.X. N-Heterocyclic carbene-Pd(II) complex derived from proline for the Mizoroki-Heck reaction in water. Tetrahedron 2011, 67, 9479-9483.

20. Liu, C.; Ni, Q.; Hu,P.; Qui, J. Oxygen-promoted $\mathrm{PdCl}_{2}$-catalyzed ligand-free Suzuki reaction in aqueous media. Org. Biomol. Chem. 2011, 9, 1054-1060.

21. Wu, X.-M.; Gu, Y.-B. TBAF-assisted palladium-catalyzed suzuki reaction in water under the ligand and base-free conditions. Lett. Org. Chem. 2012, 9, 396-400.

22. Chanthavong, F.; Leadbeater, N.E. The application of organic bases in microwave-promoted Suzuki coupling reactions in water. Tetrahedron Lett. 2006, 47, 1909-1912.

23. Öhrngren, P.; Fardost, A.; Russo, F.; Schanche, J.-S.; Fagrell, M.; Larhed, M. Evaluation of a nonresonant microwave applicator for continuous-flow chemistry applications. Org. Process Res. Dev. 2012, 16, 1053-1063.

24. Larhed, M.; Hallberg, A. Microwave-assisted high speed chemistry: A new technique in drug discovery. Drug Discov. Today 2001, 6, 406-416.

25. Kappe, C.O.; Dallinger, D. The impact of microwave synthesis on drug discovery. Nat. Rev. Drug Discov. 2006, 5, 51-63.

26. Kappe, C.O. Controlled microwave heating in modern organic synthesis. Angew. Chem. Int. Ed. 2004, 43, 6250-6284.

27. Avery, K.B.; Devine, W.G.; Kormos, C.M.; Leadbeater, N.E. Use of a silicon carbide multi-well plate in conjunction with microwave heating for rapid ligand synthesis, formation of palladium complexes, and catalyst screening in a Suzuki coupling. Tetrahedron Lett. 2009, 50, 2851-2853.

28. Prokopciva, H.; Ramirez, J.; Fernandez, E.; Kappe, C.O. Microwave-assisted one-pot diboration/Suzuki cross-couplings. A rapid route to tetrasubstituted alkenes. Tetrahedron Lett. 2008, 49, 4831-4835.

29. Glasnov, T.N.; Findenig, S.; Kappe, C.O. Heterogeneous versus homogeneous palladium catalysts for ligandless Mizoroki-Heck reactions: A comparison of batch/microwave and continuous-flow processing. Chem. Eur. J. 2009, 15, 1001-1010.

30. Leadbeater, N.E.; Marco, M. Ligand-free palladium catalysis of the Suzuki reaction in water using microwave heating. Org. Lett. 2002, 4, 2973-2976.

31. Arvela, R.K.; Leadbeater, N.E. Suzuki coupling of aryl chlorides with phenylboronic acid in water, using microwave heating with simultaneous cooling. Org. Lett. 2005, 7, 2101-2104.

32. Appukkuttan, P.; van der Eycken, E. Recent developments in microwave-assisted, transitionmetal-catalysed $\mathrm{C}-\mathrm{C}$ and $\mathrm{C}-\mathrm{N}$ bond-forming reactions. Eur. J. Org. Chem. 2008, 1133-1155.

33. Dawood, K.M. Microwave-assisted Suzuki-Miyaura and Heck-Mizoroki cross-coupling reactions of aryl chlorides and bromides in water using stable benzothiazole-based palladium(II) precatalysts. Tetrahedron 2007, 63, 9642-9651.

34. Larhed, M.; Hallberg, A. Microwave-promoted palladium-catalyzed coupling reactions. J. Org. Chem. 1996, 61, 9582-9584. 
35. Nilsson, P.; Ofsson, K.; Larhed, M. Microwave-assisted and metal-catalyzed coupling reactions. Top. Curr. Chem. 2006, 266, 103-144.

36. Yılmaz, Ü.; Şireci, N.; Deniz, S.; Küçükbay, H. Synthesis and microwave-assisted catalytic activity of novel bis-benzimidazole salts bearing furfuryl and thenyl moieties in Heck and Suzuki cross-coupling reactions. Appl. Organomet. Chem. 2010, 24, 414-420.

37. Küçükbay, H.; Şireci, N.; Yılmaz, Ü.; Akkurt, M.; Yalçın, Ş.P.; Tahir, M.N.; Ott, H. Synthesis, characterization and microwave-assisted catalytic activity of novel benzimidazole salts bearing piperidine and morpholine moieties in Heck cross-coupling reactions. Appl. Organomet. Chem. 2011, 25, 255-261.

38. Yılmaz, Ü.; Küçükbay, H.; Şireci, N.; Akkurt, M.; Günal, S.; Durmaz, R.; Tahir, M.N. Synthesis, microwave-promoted catalytic activity in Suzuki-Miyaura cross-coupling reactions and antimicrobial properties of novel benzimidazole salts bearing trimethylsilyl group. Appl. Organomet. Chem. 2011, 25, 366-373.

39. Şireci, N.; Yılmaz, Ü.; Küçükbay, H. Microwave assisted catalytic activity of some bis-5(6)-nitrobenzimidazole salts for Heck and Suzuki cross-coupling reactions. Asian J. Chem. 2010, 22, 7153-7158.

40. Küçükbay, H.; Şireci, N.; Yılmaz, Ü.; Deniz, S.; Akkurt, M.; Baktır, Z.; Büyükgüngör, O. Synthesis, characterization, and microwave-promoted catalytic activity of novel benzimidazole salts bearing silicon-containing substituents in Heck-Mizoroki and Suzuki-Miyaura cross-coupling reactions under aerobic conditions. Turk. J. Chem. 2012, 36, 201-217.

41. Phillips, M.A. The formation of 2-substituted benzimidazoles. J. Chem. Soc. 1928, 13, 2393-2399.

42. Şireci, N; Yılmaz, Ü.; Küçükbay, H.; Akkurt, M.; Baktır, Z.; Türktekin, S.; Büyükgüngör, O. Synthesis of 1 -substituted benzimidazole metal complexes and structural characterization of dichlorobis(1-phenyl-1H-benzimidazole- $\left.\mathrm{KN}^{3}\right)$ cobalt(II) and dichlorobis(1-phenyl-1H-benzimidazole$\kappa \mathrm{N}^{3}$ )zinc(II). J. Coord. Chem. 2011, 64, 1894-1902.

43. Çetinkaya, E.; Hitchcock, P.B.; Küçükbay, H.; Lappert, M.F.; Al-Juaid, S. XXIV*. Preparation of two enetetramine-derived carbenerhodium(I) chloride complexes $\operatorname{RhCl}\left(\mathrm{L}^{\mathrm{R}}\right)_{3}$ and $\left[\mathrm{RhCl}(\mathrm{COD}) \mathrm{L}^{\mathrm{R}}\right]\left\{\mathrm{LR}=\mathrm{CN}(\mathrm{Me})\left((\mathrm{CH})_{4} \mathrm{CNMe}-o\right\}\right.$ and the preparation and X-ray structures of the enetetramine $\mathrm{L} 2 \mathrm{R}$ and its salt $\left[\mathrm{L}_{2}{ }^{\mathrm{R}}\right]\left[\mathrm{BF}_{4}\right]_{2}$. J. Organomet. Chem. 1994, 481, 89-95.

44. Çetinkaya, B.; Çetinkaya, E.; Chamizo, J.A.; Hitchcock, P.B.; Jasim, H.A.; Küçükbay, H.; Lappert, M.F. Synthesis and structures of 1,3,1',3'-tetrabenzyl-2,2'-biimidazolidinylidenes (electron-rich alkenes), their aminal intermediates and their degradation products. J. Chem. Soc. Perkin. Trans. 1 1998, 2047-2054.

45. Yılmaz, Ü.; Küçükbay, H. Synthis and properties of new endotricyclic electron-rich olefins and their some derivatives. Asian J. Chem. 2009, 21, 6149-6155.

46. Rosa, G.R.; Rosa, D.S. NCP pincer palladacycle as a phosphine-free catalyst precursor for the Heck-Mizoroki coupling of aryl halides. RSC Adv. 2012, 2, 5080-5083.

47. Higgs, G. Novel treatments for asthma. Chem. Ind. 1997, 827-830.

48. De Vries, J.G. The Heck reaction in the production of fine chemicals. Can. J. Chem. 2001, 79, 1086-1092.

49. Tang, S.; Li, S.-H.; Yan, W.-B. Palladium-catalyzed cross-coupling reaction of aryl(trialkyl)silanes with aryl nitrile. Tetrahedron Lett. 2012, 53, 6743-6746. 
50. Nolan, S.P. N-Heterocyclic Carbenes in Synthesis; Wiley-VCH: Weinheim, Germany, 2006; pp. 55-70.

51. Huang, W.; Guo, J.; Xiao, Y.; Zhu, M.; Zou, G.; Tang, J. Palladium-benzimidazolium salt catalyst systems for Suzuki coupling: Development of a practical and highly active palladium catalyst system for coupling of aromatic halides with arylboronic acids. Tetrahedron 2005, 61, 9783-9790.

52. Gök, Y.; Gürbüz, N.; Özdemir, İ.; Çetinkaya, B.; Çetinkaya, E. Benzimidazolin-2-ylidenepalladium-catalysed coupling reactions of aryl halides. Appl. Organomet. Chem. 2005, 19, 870-874.

53. Singh, R.; Viciu, M.S.; Kramareva, N.; Navarro, O.; Nolan, S.P. Simple (imidazol-2-ylidene)-Pdacetate complexes as effective precatalysts for sterically hindered Suzuki-Miyaura couplings. Org. Lett. 2005, 7, 1829-1832.

54. Li, H.Y.; Cai, C. A novel system for the Suzuki cross-coupling reaction catalyzed with light fluorous palladium-NHC-complex. J. Fluorine Chem. 2012, 144, 143-146.

55. Borja, G.; Monge-Marcet, A.; Pleixats, R.; Parella, T.; Cattoen, X.; Man, M.M.C. Recyclable hybrid silica-based catalysts derived from Pd-NHC complexes for Suzuki, Heck, Sonogashira reactions. Eur. J. Org. Chem. 2012, 19, 3625-3635.

56. Lee, J.-Y.; Cheng, P.-Y.; Tsai, Y.-H.; Lin, G.-R.; Liu, S.-P.; Sie, M.-H.; Lee, H.M. efficient heck reactions catalyzed by Palladium(0) and -(II) complexes bearing $N$-heterocyclic carbene and amide functionalities. Organometallics 2010, 29, 3901-3911.

57. Durap, F.; Metin, Ö.; Aydemir, M.; Özkar, S. New route to synthesis of PVP-stabilized palladium(0) nanoclusters and their enhanced catalytic activity in Heck and Suzuki cross-coupling reactions. Appl. Organomet. Chem. 2009, 23, 498-503.

58. Akba, O.; Durap, F.; Aydemir, M.; Baysal, A.; Gümgüm, B.; Özkar, S. Synthesis and characterizations of $N, N, N^{\prime}, N^{\prime}$-tetrakis (diphenylphosphino)ethylendiamine derivatives: Use of palladium(II) complex as pre-catalyst in Suzuki coupling and Heck reactions. J. Organomet. Chem. 2009, 694, 731-736.

59. Xi, C.; Wu, Y.; Yan, X. cis-Fashioned palladium (II) complexes of 2-phenylbenzimidazole ligands: Synthesis, characterization, and catalytic behavior towards Suzuki-Miyaura reaction. J. Organomet. Chem. 2008, 693, 3842-3846.

Sample Availability: Samples of the all compounds are available from the authors.

(C) 2013 by the authors; licensee MDPI, Basel, Switzerland. This article is an open access article distributed under the terms and conditions of the Creative Commons Attribution license (http://creativecommons.org/licenses/by/3.0/). 